Proceedings of XIX International Scientific Conference "New Technologies and Achievements in Metallurgy, Material Engineering, Production Engineering and Physics", Częstochowa, Poland, June 7-8, 2018

\title{
Pulsed Nonlinear Coupler as an Effective Tool for the Bell-Like States Generation
}

\author{
J.K. Kalaga ${ }^{a}$, M.W. JARosik ${ }^{b}$, R. Szczęśniak ${ }^{b}$, Thi Dung NGuYen ${ }^{a}$ And W. Leoński ${ }^{a *}$ \\ ${ }^{a}$ Quantum Optics and Engineering Division, Institute of Physics, University of Zielona Góra, \\ Prof. Z. Szafrana 4a, 65-516 Zielona Góra, Poland \\ ${ }^{b}$ Institute of Physics, Częstochowa University of Technology, al. Armii Krajowej 19, 42-200 Częstochowa, Poland
}

\begin{abstract}
We discuss a model consisting of two nonlinear oscillators, where a train of periodic ultrashort laser pulses excites one of them. We show that the system can behave as a two-qubit system and produce maximally entangled Bell states. Such generation is more effective than that for the system with continuous excitation.
\end{abstract}

DOI: 10.12693/APhysPolA.135.273

PACS/topics: 42.65.Hw, 03.65.Aa, 03.65.Ud

\section{Introduction}

The preparation and engineering of nonclassical quantum states became one of the most important topics in the research related to quantum optics and quantum information theory. The special role plays such schemes of the state engineering which lead to the generation of the states defined in finite-dimensional Hilbert spaces. In the 90s' Pegg and Barnet have proposed the schemes and systems allowing to generate optical qubits. Such systems, based on linear optical elements, are called linear quantum scissors [1,2]. From the other side, systems comprising nonlinear elements, (in particular, the systems with nonlinear Kerr media) were considered as a potential tool for optical state truncation [3-5], and were called nonlinear quantum scissors.

In this paper, we study a model of two mutually interacting nonlinear oscillators where one of them is excited by a train of ultrashort coherent pulses. For such system, we show that under some special conditions, its evolution remains closed within a finite set of $n$-photon states and can be treated as the nonlinear quantum scissors. Additionally, discussed here model could be a source of the maximally entangled Bell states. Such generation of the Bell states is more efficient than that appearing in the analogous system but excited by a continuous external field.

\section{The model}

We consider a model of a Kerr-like coupler containing two quantum nonlinear oscillators. The oscillators couple each other the continuous linear interaction and additionally, one of them is excited by a series of ultrashort external coherent pulses. Our system is governed by the following Hamiltonian:

*corresponding author; e-mail: W.Leonski@if.uz.zgora.pl

$$
\hat{H}=\hat{H}_{N L}+\hat{H}_{K},
$$

where $H_{N L}$ describes two nonlinear oscillators and the interaction between them

$$
\begin{gathered}
\hat{H}_{N L}=\frac{\chi_{a}}{2}\left(\hat{a}^{\dagger}\right)^{2} \hat{a}^{2}+\frac{\chi_{b}}{2}\left(\hat{b}^{\dagger}\right)^{2} \hat{b}^{2} \\
+\epsilon \hat{a}^{\dagger} \hat{b}+\epsilon^{*} \hat{a} \hat{b}^{\dagger}+\chi_{a b} \hat{a}^{\dagger} \hat{a} \hat{b}^{\dagger} \hat{b}
\end{gathered}
$$

whereas

$$
\hat{H}_{K}=\sum_{n=0}^{\infty} \delta(t-n T)\left(\alpha \hat{a}^{\dagger}+\alpha^{*} \hat{a}\right)
$$

governs the interaction with the external field which is modeled by the sum of the Dirac-delta functions. The operators $\hat{a}^{\dagger}\left(\hat{b}^{\dagger}\right)$ and $\hat{a}(\hat{b})$ are the bosonic creation (annihilation) operators corresponding to the two modes labelled by $a$ and $b$. The oscillators are characterized by the nonlinear parameters $\chi_{a}$ and $\chi_{b}$, which correspond to the third-order susceptibility of the Kerr medium in nonlinear optical systems. The parameter $\epsilon$ is the strength of the linear coupling between two modes and $\chi_{a b}$ describes the cross-action process. We assume here that $\chi_{a}=\chi_{b}=\chi_{a b}=\chi$. The strength of the excitation by the pulses is described by $\alpha$, the time $T$ denotes a period of time between two subsequent pulses and $n$ labels the number of the external pulse.

In our considerations, we assume that the parameters $\epsilon=\epsilon^{*}, \alpha=\alpha^{*}$ are much smaller than those describing the nonlinearities $(\alpha, \epsilon \ll \chi)$. Thanks to those assumptions the system behaves as the nonlinear quantum scissors [3-5] - evolution is closed within a finite set of the $n$-photon states. Additionally, when initially the system is in the vacuum state $|00\rangle$, its evolution is practically limited to the three states. In consequence, the truncated wave-function can be expressed as

$$
|\psi(n)\rangle_{\text {cut }}=C_{00}|00\rangle+C_{01}|01\rangle+C_{10}|10\rangle
$$

where $C_{i j}$ are complex probability amplitudes related to the states $|i j\rangle$. What is interesting, when we consider the system involving the cross-coupling, the state $|11\rangle$ is not populated contrary to the situation when the cross-Kerr interaction is absent [6]. 
Applying the standard procedure [7], we perturbatively derive the following set of equations for the probability amplitudes which are relevant for the system's evolution:

$$
\begin{aligned}
& C_{00}(n)=\frac{1}{\Omega^{2}}\left(\epsilon^{2} T^{2}+\alpha^{2} \cos (n \Omega)\right), \\
& C_{01}(n)=\frac{\alpha \epsilon T}{\Omega^{2}}(-1+\cos (n \Omega)) \\
& C_{10}(n)=-\mathrm{i} \frac{\alpha}{\Omega} \sin (n \Omega),
\end{aligned}
$$

where $\Omega=\sqrt{\alpha^{2}+\epsilon^{2} T^{2}}$ is a frequency which appears in our system. The time-evolution of the probabilities corresponding to the three states appearing in the truncated wave-function is shown in Fig. 1. We have assumed here that the time between two succeeding pulses $T=\pi$, whereas the other parameters are: $\chi_{a}=\chi_{b}=\chi_{a b}=1$, $\epsilon=1 / 100$ and $\alpha=1 / 25$.

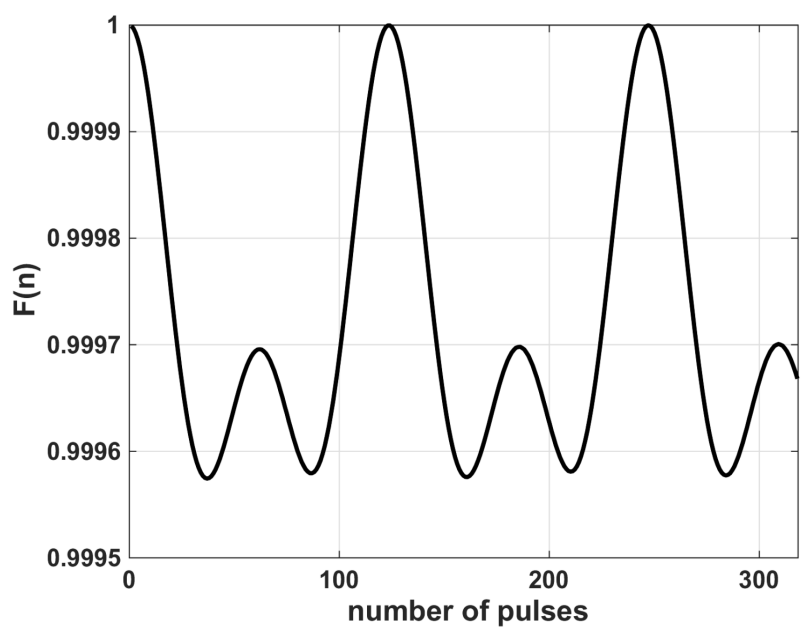

Fig. 1. The time-evolution of the probabilities $P_{i j}=\left|C_{i j}\right|^{2}$.

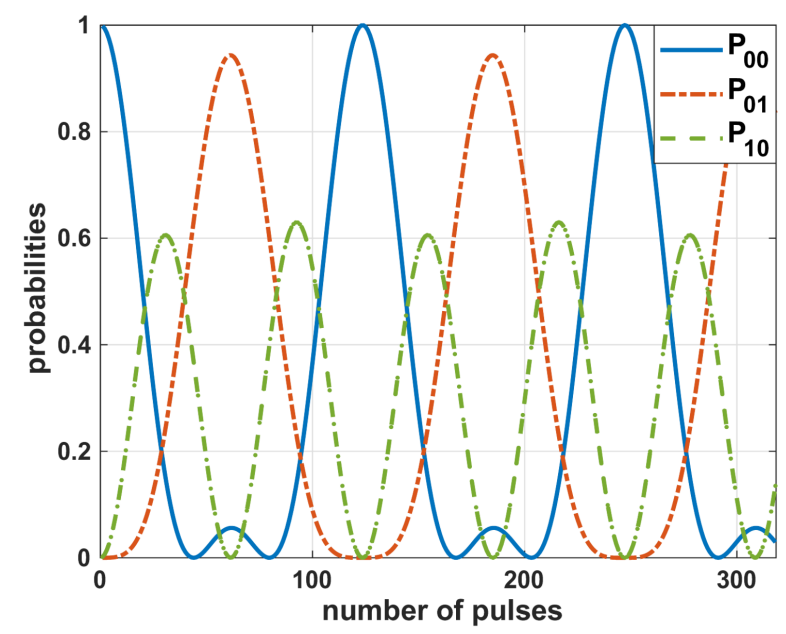

Fig. 2. Time evolution of the parameter $F(n)$.

To verify the exactness of our approximate analytical solution we apply the fidelity between the two wave functions

$$
F(n)=\left|\langle\psi(n) \mid \psi(n)\rangle_{\text {cut }}\right|,
$$

where $|\psi\rangle_{\text {cut }}$ is derived from the analytical formulae, whereas $|\psi\rangle$ was found with application of the "exact" numerical methods. At this point, it should be mentioned that in the papers devoted to the solid state physics the fidelity is called the Loschmidt echo [8, 9]. Thus, in Fig. 2 we show the time-evolution of the fidelity. We see here that $F(n)$ reaches the values which are close to the unity. That means that our quantum scissors approximation works very well and our system behaves as the qubit-qubit system.

\section{The entanglement}

In our study, we concentrate on the possibility of generation of the entangled states by the Kerr-like coupler when the cross-Kerr interaction is present.

As a measure of the entanglement, we apply the negativity $N(\hat{\rho})$ which is based on the negative partial transposition (NPT) criterion [10,11]. $N(\hat{\rho})$ is determined by the eigenvalues $\lambda_{i}$ of the partially transposed density matrix $\hat{\rho}^{T}$ and can be written as

$$
N(\hat{\rho})=\max \left(0,-2 \min \lambda_{i}\right),
$$

where we truncate the density matrix to the subspace $2 \otimes 2\left(\hat{\rho}=|\psi\rangle_{\text {cutcut }}\langle\psi|\right)$. The negativity takes zero value for all separable states, and is equal to the unity for the maximally entangled states.

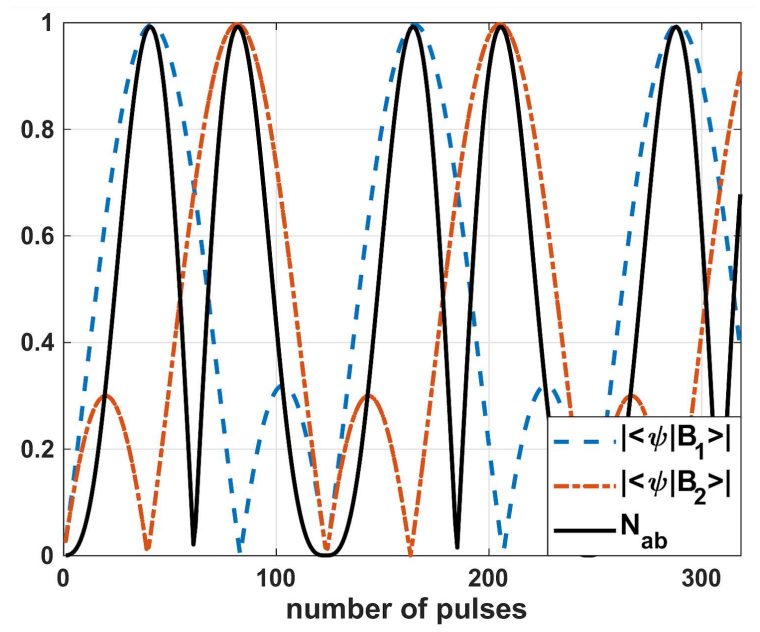

Fig. 3. The time-evolution of the negativity $N_{a b}$ and the fidelities corresponding to the states $\left|B_{1}\right\rangle$ and $\left|B_{2}\right\rangle$.

In Fig. 3 we see that the negativity reaches the values from zero to unity and thus, the system can lead to the generation of the maximally entangled states. The moments of time corresponding to such generation are the same as those for which the probabilities $P_{01} \approx P_{10} \approx 1 / 2$ (see Figs. 1 and 3 ). At the same times, the probability $P_{00}$ becomes close to zero. Therefore, we can conclude that maximally entangled states produced in the system are the Bell states 


$$
\begin{aligned}
& \left|B_{1}\right\rangle=\frac{1}{\sqrt{2}}(|01\rangle+\mathrm{i}|10\rangle), \\
& \left|B_{2}\right\rangle=\frac{1}{\sqrt{2}}(|10\rangle+\mathrm{i}|01\rangle) .
\end{aligned}
$$

The probabilities of finding $\left|B_{1}\right\rangle$ and $\left|B_{2}\right\rangle$ are plotted in Fig. 3. We see that the maximal values of the probabilities for the Bell states reach the values equal to the unity at some moments of time. Additionally, the Bell states $\left|B_{1}\right\rangle$ and $\left|B_{2}\right\rangle$ appear alternately.

What is interesting, the system excited by ultrashort pulses produces entanglement states more effectively than that discussed in [12] and excited by the field characterized by the continuous constant amplitude. For the system considered in [12], the maximal values of the negativity were equal to $\approx 0.6$. Additionally, when we take into account the cross-coupling interaction, the Bell states (8) are produced with higher probabilities than for the cases when such coupling is neglected. Thus, we see that the cross-coupling processes are responsible for the higher efficiency and the periodicity of generation of the maximally entangled states.

\section{Conclusion}

In the present paper, the model of two mutually interacting quantum nonlinear oscillators (Kerr-like coupler) excited by a series of ultrashort external coherent pulses was considered. We have shown here that when both: external excitation and the oscillator-oscillator interaction are weak, our system can be treated as the nonlinear quantum scissors. In consequence, it behaves as the qubit-qubit system. Moreover, the proposed model can be applied as a potential source of the maximally entangled states (the Bell states). What is relevant, the model of the Kerr-like coupler considered here is able to generate maximally entangled states more efficiently than other systems already discussed in other papers. For instance, our model seems to be more efficient in the generation of Bell states than the Kerr-like coupler without the cross-Kerr interaction, and the coupler continuously excited by an external field with a constant envelope.

\section{References}

[1] D.T. Pegg, L.S. Phillips, S.M. Barnett, Phys. Rev. Lett. 81, 1604 (1998).

[2] S.M. Barnett, D.T. Pegg, Phys. Rev. A 60, 4965 (1999).

[3] W. Leoński, R. Tanaś, Phys. Rev. A 49, R20 (1994).

[4] W. Leoński, Phys. Rev. A 55, 3874 (1997).

[5] W. Leoński, A. Kowalewska-Kudłaszyk, in: Progress in Optics, Ed. E. Wolf, Vol. 56, Elsevier, 2011, p. 131.

[6] A. Kowalewska-Kudłaszyk, W. Leoński, Thi Dung Nguyen, Van Cao Long, Phys. Scr. 160, 014023 (2014).

[7] W. Leoński, S. Dyrting, R. Tanaś, J. Mod. Opt. 44, 2105 (1997)

[8] D. Woźniak, A. Drzewiński, G. Kamieniarz, Acta Phys. Pol. A 127, 333 (2015).

[9] D. Woźniak, A. Drzewiński, G. Kamieniarz, Acta Phys. Pol. A 130, 1395 (2016).

[10] A. Peres, Phys. Rev. Lett. 77, 1413 (1996).

[11] M. Horodecki, P. Horodecki, M. Horodecki, R. Horodecki, Phys. Lett. A 223, 1 (1996).

[12] A. Kowalewska-Kudłaszyk, Phys. Scr. 153, 014039 (2013). 